\title{
Off-axis optical scanning holography [Invited]
}

\author{
Yaping Zhang, ${ }^{1,5}$ (1) Yongwei YaO, ${ }^{1}$ Jingyuan Zhang, ${ }^{1}$ Jung-Ping LiU, ${ }^{2,3,6}$ (1) AND \\ Ting-Chung Poon ${ }^{2,4}$ \\ ${ }^{1}$ Yunnan Provincial Key Laboratory of Modern Information Optics, Kunming University of Science and Technology, Kunming, \\ Yunnan 650500, China \\ ${ }^{2}$ Department of Photonics, Feng Chia University, 100 Wenhwa Rd., Seatwen, Taichung 40724, Taiwan \\ ${ }^{3}$ Digital Optics Center, Feng Chia University, 100 Wenhwa Rd., Seatwen, Taichung 40724, Taiwan \\ ${ }^{4}$ Bradley Department of Electrical and Computer Engineering, Virginia Tech, Blacksburg, Virginia 24061, USA \\ ${ }^{5}$ e-mail: yaping.zhang@gmail.com \\ ${ }^{6}$ e-mail: jpliu@fcu.edu.tw
}

Received 1 October 2021; revised 20 November 2021; accepted 26 November 2021; posted 2 December 2021; published 21 December 2021

\begin{abstract}
Optical scanning holography (OSH) involves the principles of optical scanning and heterodyning. The use of heterodyning leads to phase-preserving, which is the basic idea of holography. While heterodyning has numerous advantages, it requires complicated and expensive electronic processing. We investigate an off-axis approach to $\mathrm{OSH}$, thereby eliminating the use of heterodyning for phase retrieval. We develop optical scanning theory for holographic imaging and show that by properly designing the scanning beam, we can perform coherent and incoherent holographic recording. Simulation results are provided to verify the proposed idea. ๑ 2021 Optica Publishing Group under the terms of the Optica Open Access Publishing Agreement
\end{abstract}

\section{INTRODUCTION}

Optical scanning holography (OSH) is a single-pixel digital holographic recording technology that is highly sophisticated with many facets and applications $[1,2]$. Some unique applications have been demonstrated already by $\mathrm{OSH}$, such as fluorescence 3D microscopy [3-5], PSF-engineered holography [6], cryptography $[7,8]$, compressive holography [9], 3D pattern recognition [10], preprocessing and sectioning in holography $[11,12]$, and most recently, the direct digital recording of a cylindrical hologram [13]. OSH is based on two principles: optical scanning and heterodyning. Optical scanning processors can provide flexibility as well as accuracy as they continue to offer practical applications compared to parallel coherent optical processors. Indeed, one can find an excellent example in scanning confocal microscopes [14]. Heterodyning can handle phase-sensitive detection, implying a phase-preserving procedure found in holography [15]. Instead of heterodyning, homodyning also has been employed in OSH [16]. Since the standard optical system of OSH employs an interferometric approach for either heterodyning or homodyning, recent efforts have been proposed to partially solve the environmentally sensitive aspect of the use of an interferometer during data acquisition [17,18]. In addition, to reduce the complexity of the optical system, electronic scanning instead of physical optical scanning has been investigated $[19,20]$. In this paper, we investigate the use of an off-axis approach to replace the use of heterodyning or homodyning for phase retrieval. Although heterodyning can reduce the noise to the shot noise limit in optical detection, it tends to complicate the electronic processing and increase the cost for the overall system. In Section 2, we present a general optical scanning theory for holographic imaging. We show that for scanning imaging, a mask in front of a photodetector will determine the coherence of the optical system. In Section 3, we show that by choosing an off-axis plane wave and an on-axis spherical wave as a scanning beam, we achieve holographic recording coherently and incoherently, depending on the size of the mask in front of the photodetector. We also show reconstruction mathematics. In Section 4, we show some simulation results to verify our proposed ideas, and finally in the last section, we offer some conclusions.

\section{GENERAL OPTICAL SCANNING THEORY FOR HOLOGRAPHIC IMAGING}

In standard OSH, a holographic information of a $3 \mathrm{D}$ object is acquired by single $2 \mathrm{D}$ scanning of the object with a combination of a plane wave at temporal frequency $\omega_{0}$ and a spherical wave at frequency $\omega_{0}+\Omega$, as illustrated in Fig. 1(a), where we assume a slide of the $3 \mathrm{D}$ object represented by the transparency function $t(x, y ; z)$, which is complex in general. The interference of the plane wave and the spherical wave gives a Fresnel zone plate pattern at the object transparency. However, due to the temporal difference of the two waves, we actually have a dynamic FZP, which is termed the time-dependent FZP [21]. Circular fringes 


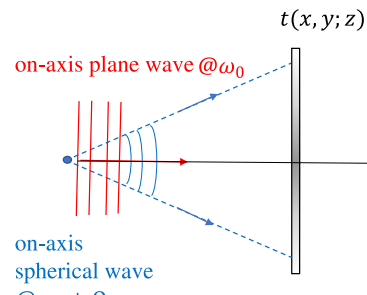

(a)

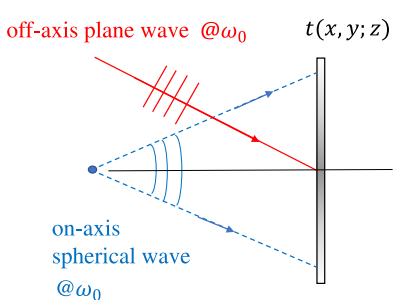

(b)
Fig. 1. Scanning patterns on object: (a) time-dependent FZP, and (b) static off-axis FZP with spatial carrier.

within the FZP move with a speed that is proportional to the temporal frequency difference $\Omega$. Photodetection of the two waves exiting the transparency at a photodetector performs heterodyning and gives a baseband current $i_{\text {base }}$ and a heterodyne current $i_{\text {het }}$ at frequency $\Omega$ [21]. The heterodyne frequency is the carrier that carries the holographic information of the scanned object. Now, in Fig. 1(b), we show our proposed offaxis approach. The plane wave and the spherical wave are now not aligned along the same direction. The off-axis plane wave at an offset angle $\theta$ causes the overall scan beam to become a static off-axis FZP as the temporal frequency of the plane wave and the spherical wave are the same. However, the static FZP is riding on a spatial carrier due to the offset angle, which would convert to a temporal carrier in the current upon scanning. The temporal frequency is $f_{t}=v f_{s}$, where $v$ is the scan speed of the scanning beam, and $f_{s}$ is the frequency of the spatial carrier. This off-axis approach to $\mathrm{OSH}$ is reminiscent of traditional holography in that the larger the offset plane wave angle, the more the separation of the twin image and the focused reconstructed image. However, in off-axis OSH, recording is done electronically and the spatial carrier is converted to temporal carrier through scanning. This is one of the main advantages of using scanning holography because a high-resolution recording medium is not required. Next, we will present a general optical scanning theory for holographic imaging and then, specifically as an example, we will discuss a situation when the scanning beam consists of a spherical wave and an off-axis plane wave, which leads to holographic recording of the scanned $3 \mathrm{D}$ object.

Figure 2 illustrates the 2D scanning system. Scanning can be performed either by moving the optical beams over the object or by moving the object situated on an $x-y$ scanning platform with a fixed optical beam. In Fig. 2, we show a plane wave and a spherical wave that are used to $2 \mathrm{D}$ scan an object transparency $t(x, y ; z)$, located $z_{0}+z$ away from the point source that generates the spherical wave. Lens $\mathrm{L}$ is a Fourier transform lens, and the mask $m(x, y)$, located at the Fourier plane of the lens and just in front of the photodetector PD, will control the size of the active area of the photodetector. The photodetector gives a scanned output in the form of a current. In general, let us assume the complex field $z+z_{0}$ away from $t(x, y ; z)$ is given by $a(x, y)$. Then the scanning beam on $t(x, y ; z)$ is $b\left(x, y ; z+z_{0}\right)$, which is the interference of the off-axis plane wave and

$$
a(x, y) * h\left(x, y ; z+z_{0}\right)
$$

with $h(x, y ; z)=\frac{j k_{0}}{2 \pi z} e^{-j k_{0} z} e^{\frac{-j k_{0}\left(x^{2}+y^{2}\right)}{2 z}}$ being the spatial impulse response in Fourier optics [15,21]. The complex field after the object slide is given by $b\left(x^{\prime}-x, y^{\prime}-y ; z+z_{0}\right) \times$ $t\left(x^{\prime}, y^{\prime} ; z\right)$. The term $b\left(x^{\prime}-x, y^{\prime}-y ; z+z_{0}\right)$ simply means that the optical beam is scanning over $t\left(x^{\prime}, y^{\prime} ; z\right)$ according to $x=x(t)=v t$ and $y=y(t)=v t$, where $v$ is the scanning velocity of the optical beam. The complex field after the object slide is then Fourier transformed onto the mask $m(x, y)$ placed in front of photodetector PD, giving the complex field in the plane of the mask as

$$
\begin{aligned}
& {\left[e^{-j \frac{k_{0} z}{2 f^{2}}\left(x_{m}^{2}+y_{m}^{2}\right)} \iint_{-\infty}^{\infty} b\left(x^{\prime}-x, y^{\prime}-y ; z+z_{0}\right)\right.} \\
& \left.t\left(x^{\prime}, y^{\prime} ; z\right) e^{\frac{j k_{0}}{f}\left(x_{m} x^{\prime}+y_{m} y^{\prime}\right)} \mathrm{d} x^{\prime} \mathrm{d} y^{\prime}\right],
\end{aligned}
$$

where $x_{m}$ and $y_{m}$ are the coordinates in the plane of the mask. This field is caused by a single object slide. For a $3 \mathrm{D}$ object, we must integrate the field over the thickness $z$ of the 3D object, giving the total field just before the mask as

$$
\begin{aligned}
& \int e^{-j \frac{k_{0} z}{2 f^{2}}\left(x_{m}^{2}+y_{m}^{2}\right)} \iint_{-\infty}^{\infty} b\left(x^{\prime}-x, y^{\prime}-y, z+z_{0}\right) \\
& t\left(x^{\prime}, y^{\prime} ; z\right) e^{\frac{j k_{0}}{f}\left(x_{m} x^{\prime}+y_{m} y^{\prime}\right)} \mathrm{d} x^{\prime} \mathrm{d} y^{\prime} \mathrm{d} z .
\end{aligned}
$$

Finally, the complex field after the mask is

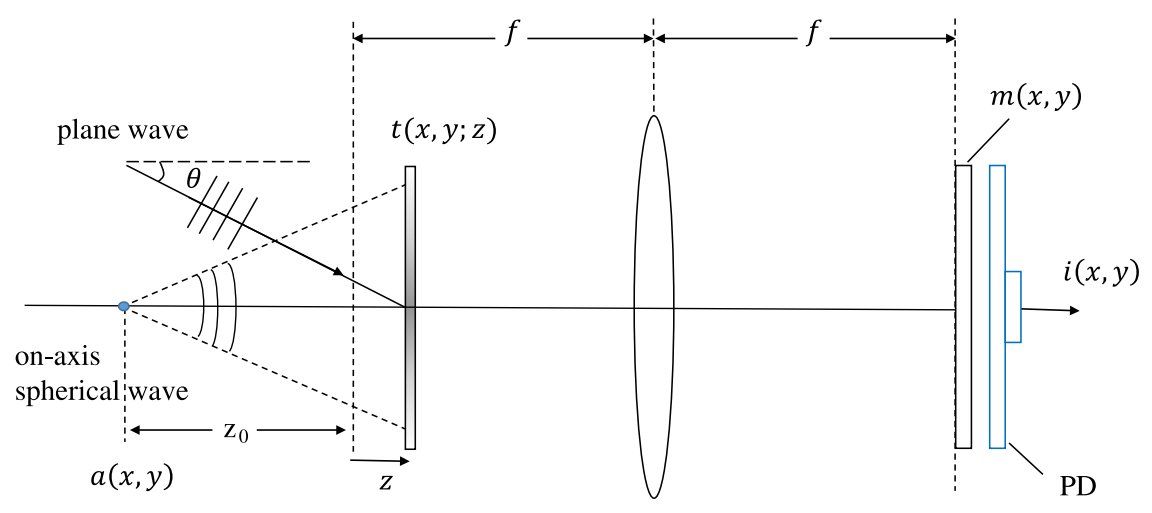

Lens L

Fig. 2. $2 \mathrm{D}$ scanning system with a scanning beam consisting of an off-axis plane wave and an on-axis spherical wave. 
$\psi\left(x, y ; x_{m}, y_{m}\right)=\left[\int e^{-j \frac{k_{0} z}{2 f^{2}}\left(x_{m}^{2}+y_{m}^{2}\right)} \iint_{-\infty}^{\infty} b\left(x^{\prime}-x, y^{\prime}-y ; z+z_{0}\right) t\left(x^{\prime}, y^{\prime} ; z\right) e^{\frac{j k_{0}}{f}\left(x_{m} x^{\prime}+y_{m} y^{\prime}\right)} \mathrm{d} x^{\prime} \mathrm{d} y^{\prime} \mathrm{d} z\right] m\left(x_{m}, y_{m}\right)$.

The photodetector responding to the intensity gives out a current $i(x, y)$ as an output by spatially integrating over the active area $D$ of the detector:

$$
\begin{aligned}
i(x, y) \propto & \iint_{D}\left|\psi\left(x, y ; x_{m}, y_{m}\right)\right|^{2} \mathrm{~d} x_{m} \mathrm{~d} y_{m} \\
= & \int\left[\int e^{-j \frac{k_{0} z^{\prime}}{2 f^{2}}\left(x_{m}{ }^{2}+y_{m}{ }^{2}\right)} \iint_{-\infty}^{\infty} b\left(x^{\prime}-x, y^{\prime}-y ; z^{\prime}+z_{0}\right) t\left(x^{\prime}, y^{\prime} ; z^{\prime}\right) e^{\frac{j k_{0}}{f}\left(x_{m} x^{\prime}+y_{m} y^{\prime}\right)} \mathrm{d} x^{\prime} \mathrm{d} y^{\prime} \mathrm{d} z^{\prime}\right] m\left(x_{m}, y_{m}\right) \\
& \times\left[\int e^{j \frac{k_{0} z^{\prime \prime}}{2 f^{2}}\left(x_{m}{ }^{2}+y_{m}{ }^{2}\right)} \iint_{-\infty}^{\infty} b^{*}\left(x^{\prime \prime}-x, y^{\prime \prime}-y ; z^{\prime \prime}+z_{0}\right) t^{*}\left(x^{\prime \prime}, y^{\prime \prime} ; z^{\prime \prime}\right) e^{\frac{-j k_{0}}{f}\left(x_{m} x^{\prime \prime}+y_{m} y^{\prime \prime}\right)} \mathrm{d} x^{\prime \prime} \mathrm{d} y^{\prime \prime} \mathrm{d} z^{\prime \prime}\right] m^{*}\left(x_{m}, y_{m}\right) \mathrm{d} x_{m} \mathrm{~d} y_{m} .
\end{aligned}
$$

By grouping all the $x_{m}$ and $y_{m}$ variables together, we can define the coherence function of the scanning system as

$$
\Gamma\left(x^{\prime}-x^{\prime \prime}, y^{\prime}-y^{\prime \prime} ; z^{\prime}-z^{\prime \prime}\right)=\int\left|m\left(x_{m}, y_{m}\right)\right|^{2} e^{j \frac{k_{0}}{f}\left[x_{m}\left(x^{\prime}-x^{\prime \prime}\right)+y_{m}\left(y^{\prime}-y^{\prime \prime}\right)\right]} e^{-j \frac{k_{0}\left(z^{\prime}-z^{\prime \prime}\right)}{2 f^{2}}\left(x_{m}^{2}+y_{m}^{2}\right)} \mathrm{d} x_{m} \mathrm{~d} y_{m} .
$$

With the definition of the coherence function, Eq. (2) becomes

$$
\begin{aligned}
i(x, y)= & \int \Gamma\left(x^{\prime}-x^{\prime \prime}, y^{\prime}-y^{\prime \prime} ; z^{\prime}-z^{\prime \prime}\right) b\left(x^{\prime}-x, y^{\prime}-y ; z^{\prime}+z_{0}\right) t\left(x^{\prime}, y^{\prime} ; z^{\prime}\right) \\
& \times b^{*}\left(x^{\prime \prime}-x, y^{\prime \prime}-y ; z^{\prime \prime}+z_{0}\right) t^{*}\left(x^{\prime \prime}, y^{\prime \prime} ; z^{\prime \prime}\right) \mathrm{d} x^{\prime} \mathrm{d} y^{\prime} \mathrm{d} z^{\prime} \mathrm{d} x^{\prime \prime} \mathrm{d} y^{\prime \prime} \mathrm{d} z^{\prime} .
\end{aligned}
$$

Thus, the equation is fairly complicated for a general situation. We shall investigate two special cases.

\section{A. Coherent Processing}

For a point detector [i.e., $|m(x, y)|^{2}=\delta(x, y)$ ], Eq. (3) becomes

$$
\Gamma\left(x^{\prime}-x^{\prime \prime}, y^{\prime}-y^{\prime \prime} ; z^{\prime}-z^{\prime \prime}\right)=\int \delta\left(x_{m}, y_{m}\right) e^{j \frac{k_{0}}{f}\left[x_{m}\left(x^{\prime}-x^{\prime \prime}\right)+y_{m}\left(y^{\prime}-y^{\prime \prime}\right)\right]} e^{-j \frac{k_{0}\left(z^{\prime}-z^{\prime \prime}\right)}{2 f^{2}}\left(x_{m}^{2}+y_{m}^{2}\right)} \mathrm{d} x_{m} \mathrm{~d} y_{m}=1
$$

With this result, Eq. (4) becomes

$$
i(x, y)=\int b\left(x^{\prime}-x, y^{\prime}-y ; z^{\prime}+z_{0}\right) t\left(x^{\prime}, y^{\prime} ; z^{\prime}\right) \times b^{*}\left(x^{\prime \prime}-x, y^{\prime \prime}-y ; z^{\prime \prime}+z_{0}\right) t^{*}\left(x^{\prime \prime}, y^{\prime \prime} ; z^{\prime \prime}\right) \mathrm{d} x^{\prime} \mathrm{d} y^{\prime} \mathrm{d} z^{\prime} \mathrm{d} x^{\prime \prime} \mathrm{d} y^{\prime \prime} \mathrm{d} z^{\prime \prime}
$$

Since the prime and double prime integrations can be performed separately, we rewrite the above equation to become

$$
\begin{aligned}
i(x, y) & =\int b\left(x^{\prime}-x, y^{\prime}-y ; z^{\prime}+z_{0}\right) t\left(x^{\prime}, y^{\prime} ; z^{\prime}\right) \mathrm{d} x^{\prime} \mathrm{d} y^{\prime} \mathrm{d} z^{\prime} \int b^{*}\left(x^{\prime \prime}-x, y^{\prime \prime}-y ; z^{\prime \prime}+z_{0}\right) t^{*}\left(x^{\prime \prime}, y^{\prime \prime} ; z^{\prime \prime}\right) \mathrm{d} x^{\prime \prime} \mathrm{d} y^{\prime \prime} \mathrm{d} z^{\prime \prime} \\
& =\left|\int b\left(x^{\prime}-x, y^{\prime}-y ; z^{\prime}+z_{0}\right) t\left(x^{\prime}, y^{\prime} ; z^{\prime}\right) \mathrm{d} x^{\prime} \mathrm{d} y^{\prime} \mathrm{d} z^{\prime}\right|^{2} \\
& =\left|\int t(x, y ; z) * b\left(-x,-y ; z+z_{0}\right) \mathrm{d} z\right|^{2}
\end{aligned}
$$

where $*$ denotes a $2 \mathrm{D}$ convolution involving $x$ and $y$ coordinates. This corresponds to coherent imaging with a coherent point spread function of the scanning system given by $b\left(-x,-y ; z+z_{0}\right)$.

\section{B. Incoherent Processing}

For an integrating detector (i.e., $\left.|m(x, y)|^{2}=1\right)$, Eq. (3) becomes

$$
\Gamma\left(x^{\prime}-x^{\prime \prime}, y^{\prime}-y^{\prime \prime} ; z^{\prime}-z^{\prime \prime}\right)=\int e^{j \frac{k_{0}}{f}\left[x_{m}\left(x^{\prime}-x^{\prime \prime}\right)+y_{m}\left(y^{\prime}-y^{\prime \prime}\right)\right]} e^{-j \frac{k_{0}\left(z^{\prime}-z^{\prime \prime}\right)}{2 f^{2}}\left(x_{m}^{2}+y_{m}^{2}\right)} \mathrm{d} x_{m} \mathrm{~d} y_{m} \sim \delta\left(x^{\prime}-x^{\prime \prime}, y^{\prime}-y^{\prime \prime} ; z^{\prime}-z^{\prime \prime}\right)
$$


because we may consider that the above integral takes the Fourier transform of a spherical wave of radius of curvature of $f^{2} /\left(z^{\prime}-z^{\prime \prime}\right)$, which can be made arbitrarily large.

With this result, Eq. (4) becomes

$$
b\left(x, y ; z+z_{0}\right)=A e^{j k_{0} \sin \theta x}+B \frac{j k_{0}}{2 \pi\left(z+z_{0}\right)} e^{\frac{-j k_{0}}{2\left(z+z_{0}\right)}\left(x^{2}+y^{2}\right)},
$$

where $\sin \theta=x_{0} / f$, and we assume $A$ and $B$ are real.

$$
\begin{aligned}
i(x, y)= & \int \delta\left(x^{\prime}-x^{\prime \prime}, y^{\prime}-y^{\prime \prime} ; z^{\prime}-z^{\prime \prime}\right) b\left(x^{\prime}-x, y^{\prime}-y ; z^{\prime}+z_{0}\right) t\left(x^{\prime}, y^{\prime} ; z^{\prime}\right) \\
& \times b^{*}\left(x^{\prime \prime}-x, y^{\prime \prime}-y, z^{\prime \prime}+z_{0}\right) t^{*}\left(x^{\prime \prime}, y^{\prime \prime} ; z^{\prime \prime}\right) \mathrm{d} x^{\prime} \mathrm{d} y^{\prime} \mathrm{d} z^{\prime} \mathrm{d} x^{\prime \prime} \mathrm{d} y^{\prime \prime} \mathrm{d} z^{\prime \prime} . \\
= & \int\left|b\left(x^{\prime}-x, y^{\prime}-y ; z^{\prime}+z_{0}\right)\right|^{2}\left|t\left(x^{\prime}, y^{\prime} ; z^{\prime}\right)\right|^{2} \mathrm{~d} x^{\prime} \mathrm{d} y^{\prime} \mathrm{d} z^{\prime} \\
= & \int|t(x, y ; z)|^{2} *\left|b\left(-x,-y ; z+z_{0}\right)\right|^{2} \mathrm{~d} z .
\end{aligned}
$$

This performs incoherent processing as it manipulates and processes the intensity distribution of the $3 \mathrm{D}$ object. $\left|b\left(-x,-y ; z+z_{0}\right)\right|^{2}$ is the intensity point spread function of the incoherent system.

We have considered extreme cases with different mask sizes. In one extreme, the mask is wide open (i.e., $|m(x, y)|^{2}=1$ ), leading to incoherent imaging; in the other extreme case, the mask is extremely small [i.e., $|m(x, y)|^{2}=\delta(x, y)$ ], leading to coherent imaging. It is not hard to envision that we have partial coherent imaging using a mask of some finite size [22,23].

\section{HOLOGRAPHIC RECORDING AND RECONSTRUCTION}

By manipulating the structure of the scanning beam $b\left(x, y ; z+z_{0}\right)$, we would achieve a holographic recording of a $3 \mathrm{D}$ object being raster scanned. Figure 3 shows a practical implementation of Fig. 2, where we have shown the location of the 3 D object.

$p_{1}(x, y)$ and $p_{2}(x, y)$ are the pupil functions, where they are located in the front focal plane of Lens L1. For $p_{1}(x, y)=A \delta\left(x-x_{0}, y\right)$ and $p_{2}(x, y)=B$, the scanning beam complex profile $b\left(x, y ; z+z_{0}\right)$ on the transparency can be modeled as the interference of an off-axis plane wave and an on-axis spherical wave shown in Fig. 3:

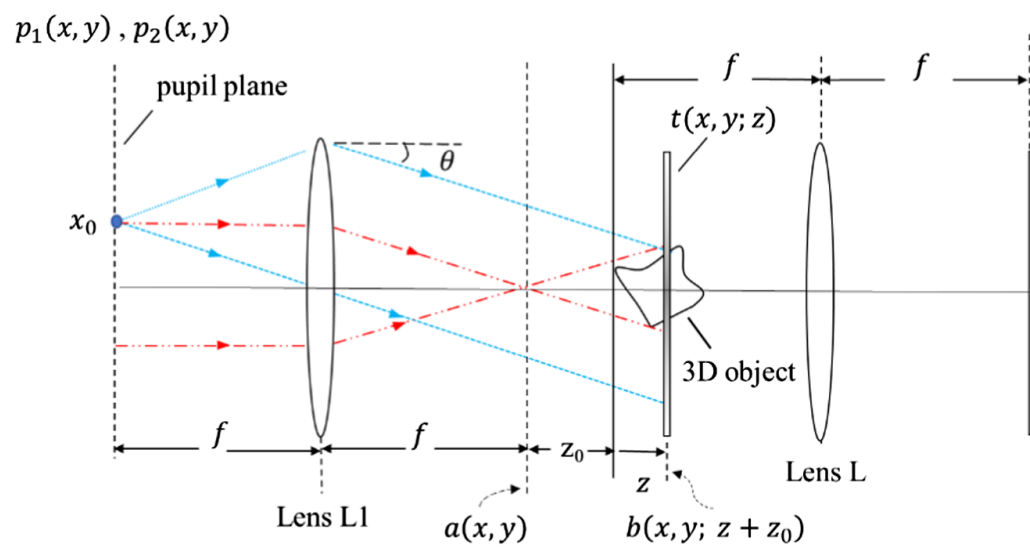

\section{A. Coherent Holographic Recording and Reconstruction}

For simplicity, let us assume there is a planar object located at $z=0$ given by $t(x, y ; z)=t_{0}(x, y) \delta(z)$. For the coherent case, from Eq. (6), we have

$$
\begin{aligned}
i(x, y) & =\left|\int t(x, y ; z) * b\left(-x,-y ; z+z_{0}\right) \mathrm{d} z\right|^{2} \\
& =\left|\int t_{0}(x, y) \delta(z) * b\left(-x,-y ; z+z_{0}\right) \mathrm{d} z\right|^{2} \\
& =\left|t_{0}(x, y) * b\left(-x,-y ; z_{0}\right)\right|^{2},
\end{aligned}
$$

where

explicitly.

Writing out Eq. (9), we have a coherent hologram, $H_{c o}(x, y)$ :

$$
\begin{aligned}
i(x, y) & =H_{c o}(x, y) \\
& =\left|t_{0}(x, y) *\left[A e^{-j k_{0} \sin \theta x}+B \frac{j k_{0}}{2 \pi z_{0}} e^{\frac{-j k_{0}}{2 z_{0}}\left(x^{2}+y^{2}\right)}\right]\right|^{2} .
\end{aligned}
$$

Fig. 3. Practical implementation of the scanning system for a $3 \mathrm{D}$ object. 
Let us evaluate the first term:

$$
\begin{aligned}
& t_{0}(x, y) * A e^{-j k_{0} \sin \theta x} \\
& =\mathcal{F}^{-1}\left\{\mathcal{F}\left\{t_{0}(x, y)\right\} \mathcal{F}\left\{A e^{-j k_{0} \sin \theta x}\right\}\right\} \\
& =\mathcal{F}^{-1}\left\{T_{0}\left(k_{x}, k_{y}\right) 4 \pi^{2} A \delta\left(k_{x}-k_{0} \sin \theta, k_{y}\right)\right\} \\
& =\mathcal{F}^{-1}\left\{T_{0}\left(k_{0} \sin \theta, 0\right) 4 \pi^{2} A \delta\left(k_{x}-k_{0} \sin \theta, k_{y}\right)\right\} \\
& =D e^{-j k_{0} \sin \theta x},
\end{aligned}
$$

where $\mathcal{F}\left\{t_{0}(x, y)\right\}=T_{0}\left(k_{x}, k_{y}\right)$ is the Fourier transform of $t_{0}(x, y)$ defined as

$$
\mathcal{F}\left\{t_{0}(x, y)\right\}=T_{0}\left(k_{x}, k_{y}\right)=\iint_{-\infty}^{\infty} t_{0}(x, y) e^{j k_{x} x+j k_{y} y} \mathrm{~d} x \mathrm{~d} y,
$$

with $k_{x}$ and $k_{y}$ being the radian spatial frequencies corresponding to the coordinates $x$ and $y$, respectively. Hence, the coherent hologram from Eq. (10) becomes

$$
\begin{aligned}
H_{c o}(x, y)= & \left|D e^{-j k_{0} \sin \theta x}+t_{0}(x, y) * B \frac{j k_{0}}{2 \pi z_{0}} e^{\frac{-j k_{0}}{2 z_{0}}\left(x^{2}+y^{2}\right)}\right|^{2} \\
= & |D|^{2}+\left|t_{0}(x, y) * B \frac{j k_{0}}{2 \pi z_{0}} e^{\frac{-j k_{0}}{2 z_{0}}\left(x^{2}+y^{2}\right)}\right|^{2} \\
& +D^{*} e^{j k_{0} \sin \theta x}\left[t_{0}(x, y) * B \frac{j k_{0}}{2 \pi z_{0}} e^{\frac{-j k_{0}}{2 z_{0}}\left(x^{2}+y^{2}\right)}\right] \\
& +D e^{-j k_{0} \sin \theta x}\left[t_{0}^{*}(x, y) * B \frac{-j k_{0}}{2 \pi z_{0}} e^{\frac{j k_{0}}{2 z_{0}}\left(x^{2}+y^{2}\right)}\right] .
\end{aligned}
$$

The coherent hologram can be written to a spatial light modulator (SLM) for optical reconstruction. For example, we can illuminate the SLM with a conjugate beam (i.e., the reconstruction plane wave is conjugate to the off-axis plane wave used to scan the object). Therefore, the conjugate beam is given by $e^{-j k_{0} \sin \theta x}$. The first two terms with the hologram equation above give rise to a zeroth-order beam upon reconstruction. The third term will give rise to a virtual image reconstruction of $t_{0}(x, y)$ at a location $z_{0}$ behind the SLM (assuming transmissive) as

$$
\begin{aligned}
e^{-j k_{0} \sin \theta x} & \times D^{*} e^{j k_{0} \sin \theta x}\left[t_{0}(x, y) * B \frac{j k_{0}}{2 \pi z_{0}} e^{\frac{-j k_{0}}{2 z_{0}}\left(x^{2}+y^{2}\right)}\right] \\
& * h^{*}\left(x, y ; z_{0}\right) \propto t_{0}(x, y) .
\end{aligned}
$$

The conjugate of $t_{0}(x, y)$ is formed at $x=z_{0} \sin \theta$ as a real image reconstruction as

$$
\begin{aligned}
e^{-j k_{0} \sin \theta x} & \times D e^{-j k_{0} \sin \theta x}\left[t_{0}^{*}(x, y) * B \frac{-j k_{0}}{2 \pi z_{0}} e^{\frac{j k_{0}}{2 z_{0}}\left(x^{2}+y^{2}\right)}\right] \\
& * h\left(x, y ; z_{0}\right) \propto e^{-j 2 k_{0} \sin \theta x} t_{0}^{*}\left(x-2 z_{0} \sin \theta, y\right) .
\end{aligned}
$$

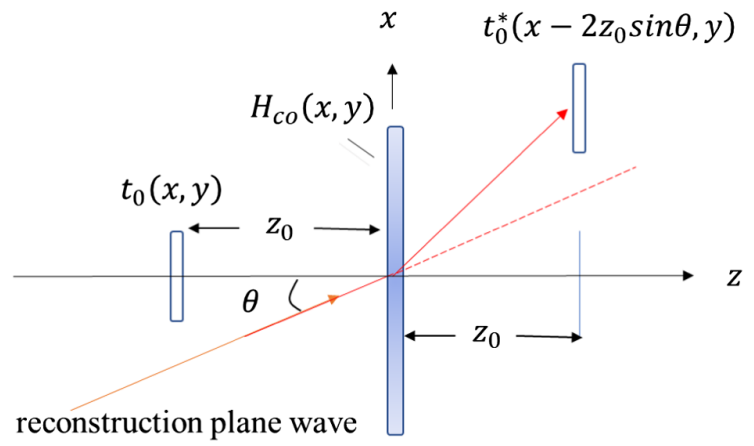

Fig. 4. Real and virtual image reconstruction from coherent hologram.

The reconstruction of the coherent hologram is shown in Fig. 4. Note that the complex amplitudes of the object are reconstructed.

\section{B. Incoherent Holographic Recording and Reconstruction}

Again, we assume $t(x, y ; z)=t_{0}(x, y) \delta(z)$. For the incoherent case, Eq. (7) becomes

$$
i(x, y)=\left|t_{0}(x, y)\right|^{2} *\left|b\left(-x,-y ; z_{0}\right)\right|^{2} .
$$

From Eq. (8), we have

$$
\begin{aligned}
\left|b\left(-x,-y ; z_{0}\right)\right|^{2}= & \left|A e^{-j k_{0} \sin \theta x}+B \frac{j k_{0}}{2 \pi z_{0}} e^{\frac{-j k_{0}}{2 z_{0}}\left(x^{2}+y^{2}\right)}\right|^{2} \\
= & |A|^{2}+\left|B \frac{k_{0}}{2 \pi z_{0}}\right|^{2}+A B \frac{k_{0}}{\pi z_{0}} \\
& \times \sin \left[\frac{k_{0}}{2 z_{0}}\left(x^{2}+y^{2}\right)-k_{0} x \sin \theta\right] \\
= & E+F \sin \left[\frac{k_{0}}{2 z_{0}}\left(x^{2}+y^{2}\right)-k_{0} x \sin \theta\right] .
\end{aligned}
$$

Substituting Eq. (15) into Eq. (14), we have an incoherent hologram, $H_{\text {inco }}(x, y)$ :

$$
\begin{aligned}
i(x, y)= & H_{\text {inco }}(x, y)=\left|t_{0}(x, y)\right|^{2} \\
& *\left\{E+F \sin \left[\frac{k_{0}}{2 z_{0}}\left(x^{2}+y^{2}\right)-k_{0} x \sin \theta\right]\right\} \\
= & G+F\left|t_{0}(x, y)\right|^{2} * \sin \left[\frac{k_{0}}{2 z_{0}}\left(x^{2}+y^{2}\right)-k_{0} x \sin \theta\right],
\end{aligned}
$$

as $\left|t_{0}(x, y)\right|^{2} * E$ gives some constant $G$. Again, let us use the conjugate beam $e^{-j k_{0} \sin \theta x}$ as a reconstruction wave if the hologram is displayed on a SLM. The first term of the hologram equation in Eq. (16) gives a zeroth-order beam. Using $\sin \vartheta=\left(e^{j \vartheta}-e^{-j \vartheta}\right) / 2 j$, the second term of the hologram equation gives rise to a real image: 


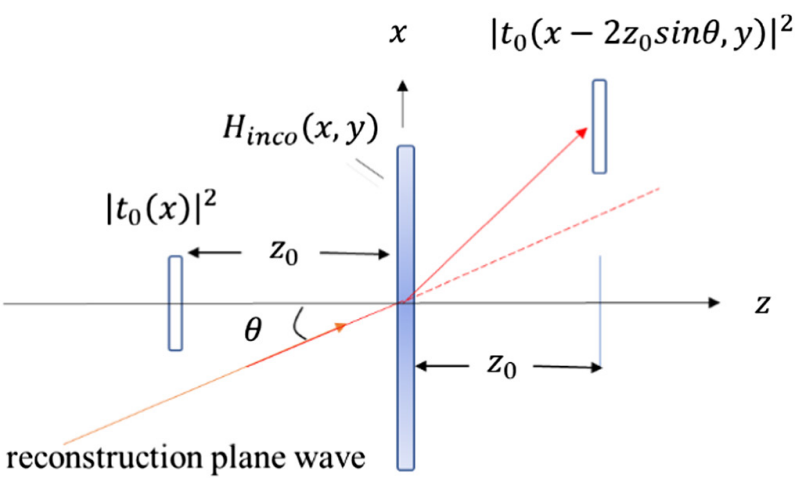

Fig. 5. Real and virtual image reconstruction for incoherent hologram.

$$
\begin{aligned}
& e^{-j k_{0} \sin \theta x}\left\{\left|t_{0}(x, y)\right|^{2} * e^{j\left[\frac{k_{0}}{2 z_{0}}\left(x^{2}+y^{2}\right)-k_{0} x \sin \theta\right]}\right\} \\
& * h\left(x, y ; z_{0}\right) \\
& \propto\left|t_{0}\left(x-2 z_{0} \sin \theta, y\right)\right|^{2} e^{-j k_{0} x \sin \theta} .
\end{aligned}
$$

The third term gives a virtual image reconstruction:

$$
\begin{aligned}
& e^{-j k_{0} \sin \theta x}\left\{\left|t_{0}(x, y)\right|^{2} * e^{-j\left[\frac{k_{0}}{2 z_{0}}\left(x^{2}+y^{2}\right)-k_{0} x \sin \theta\right]}\right\} \\
& * h^{*}\left(x, y ; z_{0}\right) \\
& \propto\left|t_{0}(x)\right|^{2} e^{-j k_{0} x \sin \theta} .
\end{aligned}
$$

The reconstruction diagram is shown in Fig. 5. Note that the intensity distributions of the object are reconstructed.

\section{SIMULATIONS RESULTS}

We present some simulations to demonstrate the coherent and incoherent processing. For coherent holographic processing, in Fig. 6(a), we show the Chinese character "Light" as a coherent object $t_{0}(x, y)$. Figure $6(\mathrm{~b})$ is the intensity plot of the coherent point spread function (CPSF) given by Eq. (8) [i.e., $\left.\left|b\left(x, y ; z+z_{0}\right)^{2}\right|\right]$. Note that the intensity of the CPSF is of the same form of the intensity point spread function (IPSF) of the incoherent case [see Eq. (15)], a property that is identical to that obtained in heterodyne OSH [23,24]. Figures 6(c) and 6(d) are the coherent hologram given by Eq. (10) and its spectrum, respectively. Figures 7 (a) and 7 (b) show the real and virtual image reconstructions of the coherent hologram calculated by Eqs. (12) and (13), respectively. Note that in these reconstructions, we observe some coherent noise from the zeroth-order beam and its twin image on the focused image planes. However, this will not be a problem if the observer is far from the reconstruction plane.

For incoherent holographic processing, in Fig. 8(a) we show the incoherent counterpart of Fig. 6. Figure 8(a) is the Chinese character "Light" as an incoherent object $\left|t_{0}(x, y)\right|^{2}$. Figure $8(\mathrm{~b})$ is the plot of the IPSF given by Eq. (15). Note that this is an intensity distribution. Figure $8(\mathrm{c})$ is the incoherent hologram calculated by Eq. (16) and its magnitude spectrum is

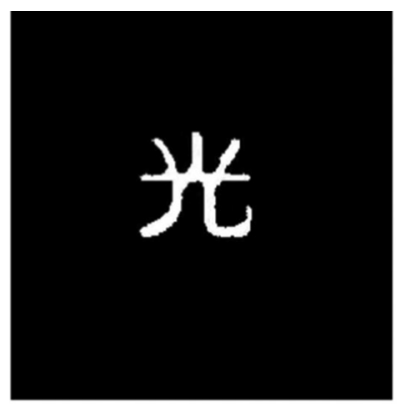

(a)

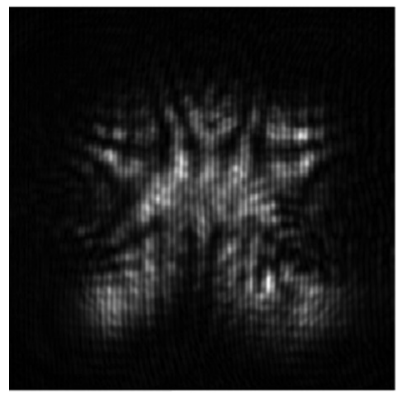

(c)

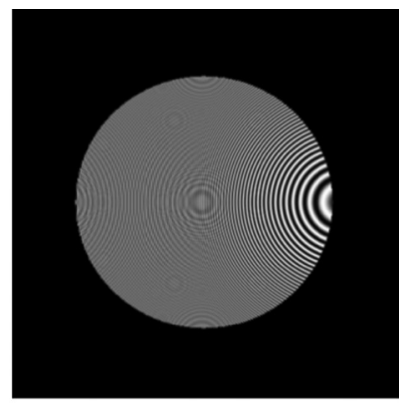

(b)

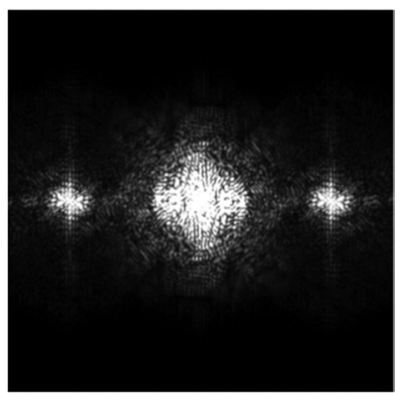

(d)
Fig. 6. (a) Original Chinese character "Light" as an coherent input object, (b) intensity plot of coherent point spread function, (c) coherent hologram of (a), and (d) magnitude spectrum of coherent hologram. In (c), we deliberately make the figure larger to observe the fine fringes in the hologram.

plotted in Fig. 8(d). Finally in Figs. 9(a) and 9(b), we show the real and virtual image reconstruction calculated by Eqs. (17) and (18), respectively, of the incoherent hologram. Interestingly, the reconstructed images are edge-enhanced, and this can be explained as follows. By inspecting the incoherent hologram given by Eq. (16), let us concentrate on the term

$$
\left|t_{0}(x, y)\right|^{2} * \sin \left[\frac{k_{0}}{2 z_{0}}\left(x^{2}+y^{2}\right)-k_{0} x \sin \theta\right] .
$$

Its spectrum is

$$
\begin{aligned}
& \mathcal{F}\left\{\left|t_{0}(x, y)\right|^{2}\right\} \mathcal{F}\left\{\sin \left[\frac{k_{0}}{2 z_{0}}\left(x^{2}+y^{2}\right)-k_{0} x \sin \theta\right]\right\} \\
& =\mathcal{F}\left\{\left|t_{0}(x, y)\right|^{2}\right\} e^{\frac{j\left[\left(k_{x}-k_{0} \sin \theta\right)^{2}+k_{y}^{2}\right] z_{0}}{2 k_{0}}}+\mathcal{F}\left\{\left|t_{0}(x, y)\right|^{2}\right\} \\
& e^{\frac{j\left[\left(k_{x}+k_{0} \sin \theta\right)^{2}+k_{y}^{2}\right] z_{0}}{2 k_{0}}} .
\end{aligned}
$$

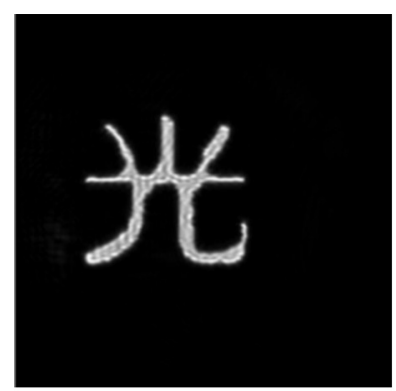

(a)

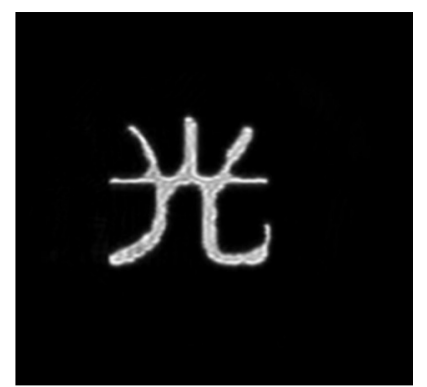

(b)
Fig. 7. (a) Real image coherent reconstruction and (b) virtual image coherent reconstruction. 


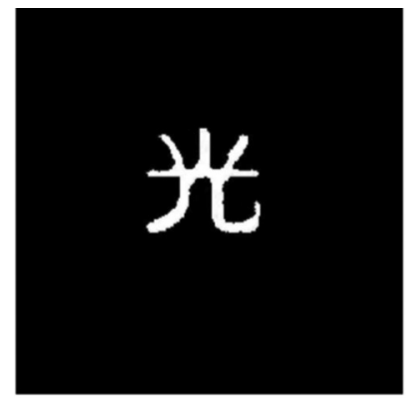

(a)

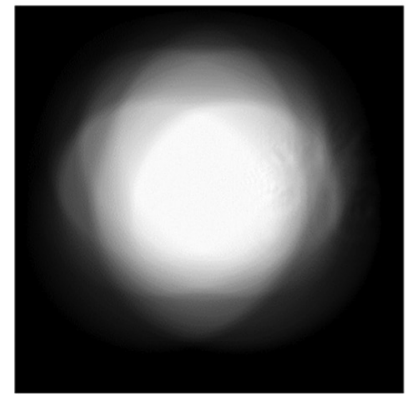

(c)

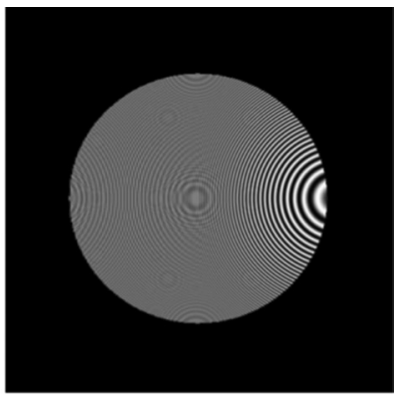

(b)

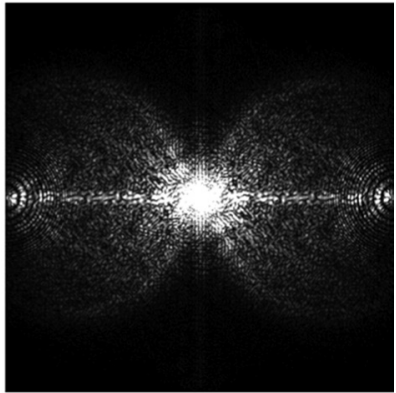

(d)
Fig. 8. (a) Original Chinese character "Light" as an incoherent input object, (b) intensity point spread function, (c) incoherent hologram of (a), and (d) magnitude spectrum of (c).

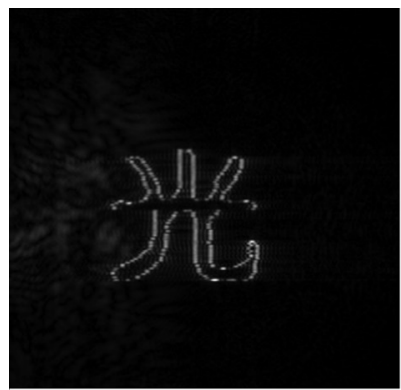

(a)

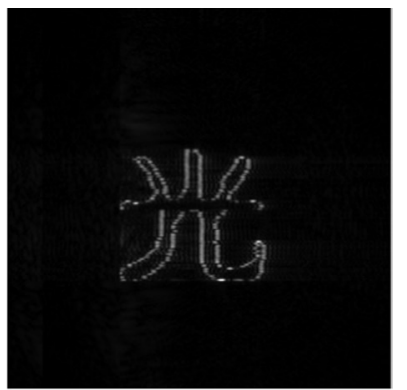

(b)
Fig. 9. (a) Real image incoherent reconstruction and (b) virtual image incoherent reconstruction.

Note that the center of $\mathcal{F}\left\{\left|t_{0}(x, y)\right|^{2}\right\}$ is at $\left(k_{x}, k_{y}\right)=(0,0)$, while the centers of the transfer functions are at $\left(k_{x}, k_{y}\right)=$ $\left( \pm k_{0} \sin \theta, 0\right)$. Because only one side of $\mathcal{F}\left\{\left|t_{0}(x, y)\right|^{2}\right\}$ overlaps with the transfer function, it accomplishes single-sided filtering and the reconstructions only retrieve the high frequency of the object, thereby exhibiting the edge enhancement illustrated in Fig. 9(a) and 9(b). This aspect of incoherent holographic processing is actually an interesting and unexpected result. For holographic imaging, we should, of course, try to recover the original image. However, edge enhancement would be important if we plan to use the reconstructed image for applications such as image recognition. We also want to point out that the achievable edge enhancement is performed only perpendicular to the axis of the plane wave tilt. This would correspond to anisotropic edge extraction in image processing.

\section{CONCLUSION}

We have developed a general theory of holographic imaging by optical scanning. By suitably designing the structured scanning beam, we can perform holographic recording of a $3 \mathrm{D}$ object. The coherence of the optical system depends on the size of the mask in front of the photodetector. For a point detector, where the mask is a pinhole, we have coherent holographic imaging. For an integrating detector, where the mask opens widely, we have incoherent holographic imaging. It is possible to perform partial coherent holographic imaging with a mask of a finite size. We have performed computer simulations to demonstrate coherent as well as incoherent holographic imaging. For incoherent holographic imaging, we have found an interesting, yet unexpected, result: edge enhancement of the original object.

Funding. National Natural Science Foundation of China (11762009, 61865007); Yunnan Provincial Science and Technology Department (2019FA025); Yunnan Provincial Program for Foreign Talent (202105AO130015); Ministry of Science and Technology, Taiwan (109-2221-E-035-076-MY3).

Disclosures. The authors declare no conflicts of interest.

Data Availability. Data underlying the results presented in this paper are not publicly available at this time but may be obtained from the authors upon reasonable request.

\section{REFERENCES}

1. T.-C. Poon, "Scanning holography and two-dimensional image processing by acousto-optic two-pupil synthesis," J. Opt. Soc. Am. A 2, 521-527 (1985).

2. W.-C. Chieh, D. S. Dilworth, E. Liu, and E. N. Leith, "Syntheticaperture chirp confocal imaging," Appl. Opt. 45, 501-510 (2006).

3. B. W. Schilling, T.-C. Poon, G. Indebetouw, B. Storrie, K. Shinoda, Y. Suzuki, and M. H. Wu, "Three-dimensional holographic fluorescence microscopy," Opt. Lett. 22, 1506-1508 (1997).

4. E. Y. Lam, X. Zhang, H. Vo, T.-C. Poon, and G. Indebetouw, "Threedimensional microscopy and sectional image reconstruction using optical scanning holography," Appl. Opt. 48, H113-H119 (2009).

5. J. Swoger, M. Martínez-Corral, J. Huisken, and E. H. K. Stelzer, "Optical scanning holography as a technique for high-resolution three-dimensional biological microscopy," J. Opt. Soc. Am. A 19, 1910-1919 (2002).

6. T.-C. Poon, "Optical scanning holography-a review of recent progress," J. Opt. Soc. Korea 13, 406-415 (2009).

7. T.-C. Poon, T. Kim, and K. Doh, "Optical scanning cryptography for secure wireless transmission," Appl. Opt. 42, 6496-6503 (2003).

8. A. Yan, Y. Wei, Z. Hu, J. Zhang, P. W. M. Tsang, and T.-C. Poon, "Optical cryptography with biometrics for multi-depth objects," Sci. Rep. 7, 12933 (2017).

9. P. W. M. Tsang, J.-P. Liu, and T.-C. Poon, "Compressive optical scanning holography," Optica 2, 476-483 (2015).

10. T. Kim and T.-C. Poon, "Optical image recognition of threedimensional objects," Appl. Opt. 38, 370-381 (1999).

11. Y. Zhang, T.-C. Poon, P. W. M. Tsang, R. Wang, and L. Wang, "Review on feature extraction for 3-D incoherent image processing using optical scanning holography, IEEE Trans. Ind. Informat. 15, 6146-6154 (2019).

12. Y. Zhang, R. Wang, P. Tsang, and T.-C. Poon, "Sectioning with edge extraction in optical incoherent imaging processing," OSA Contin. 3, 698-708 (2020).

13. J.-P. Liu, W.-T. Chen, H.-H. Wen, and T.-C. Poon, "Recording of a curved digital hologram for orthoscopic real image reconstruction," Opt. Lett. 45, 4353-4356 (2020).

14. T. Wilson and C. Sheppard, Theory and Practice of Optical Scanning Microscopy (Academic, 1984). 
15. T.-C. Poon and T. Kim, Engineering Optics with MATLAB, 2nd ed. (World Scientific, 2018), p. 242.

16. J. Rosen, G. Indebetouw, and G. Brooker, "Homodyne scanning holography," Opt. Express 14, 4280-4285 (2006).

17. T. Kim and T. Kim, "Coaxial scanning holography," Opt. Lett. 45, 2046-2049 (2020).

18. C.-M. Tsai, H.-Y. Sie, T.-C. Poon, and J.-P. Liu, "Optical scanning holography with a polarization directed flat lens," Appl. Opt. 60, B113-B118 (2021).

19. N. Yoneda, Y. Saita, and T. Nomura, "Motionless optical scanning holography," Opt. Lett. 45, 3184-3187 (2020).

20. N. Yoneda, Y. Saita, and T. Nomura, "Spatially divided phase-shifting motionless optical scanning holography," OSA Contin. 3, 3523-3535 (2020).
21. T.-C. Poon, Optical Scanning Holography with MATLAB (Springer, 2007), p. 102

22. J.-P. Liu, C.-H. Guo, W.-J. Hsiao, T.-C. Poon, and P. W. M. Tsang, "Coherence experiments in single-pixel digital holography," Opt. Lett. 40, 2366-2369 (2015).

23. J.-P. Liu, "Spatial coherence analysis for optical scanning holography," Appl. Opt. 54, A59-A66 (2015).

24. T.-C. Poon and G. Indebetouw, "Three-dimensional point spread functions of an optical heterodyne scanning image processor," Appl. Opt. 42, 1485-1492 (2003). 